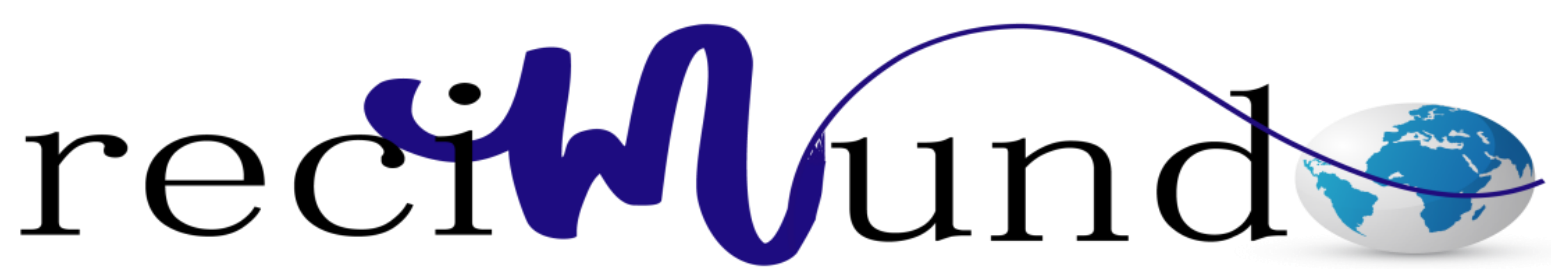

Revista Científica Mundo de la Investigación y el Conocimiento

Joao Andres Rodríguez Molina a; Pedro Javier Chong Cevallos ${ }^{\text {b}}$; Julio César Tixe Peralta ${ }^{c}$; Rogelio Andrés Leyton Acuña ${ }^{d}$

Escala de Silverman en la dificultad respiratoria neonatal

Silverman's scale in neonatal respiratory distress

Revista Científica Mundo de la Investigación y el Conocimiento. Vol. 3 núm.3. Esp., noviembre, ISSN: 2588-073X, 2019, pp. 113-127

DOI: $\underline{\text { 10.26820/recimundo/3.(3.Esp).noviembre.2019.113-127 }}$

URL: http://recimundo.com/index.php/es/article/view/601

Código UNESCO: 3205 Medicina Interna

Tipo de Investigación: Artículo de Revisión

(C) RECIMUNDO; Editorial Saberes del Conocimiento, 2019

Recibido: 15/09/2019

Aceptado: 23/10/2019

Publicado: 30/11/2019

Correspondencia: joao.andrés.rod@gmail.com

a. Médico; Investigador Independiente; Guayaquil, Ecuador; joao.andrés.rod@ gmail.com

b. Médico; Investigador Independiente; Guayaquil, Ecuador; pedrochongc@ gmail.com

c. Médico: Investigador Independiente; Guayaquil, Ecuador; julianotixe@ hotmail.com

d. Médico; Investigador Independiente; Guayaquil, Ecuador; rogleyton@ gmail.com 


\section{Escala de Silverman en la dificultad respiratoria neonatal}

Vol. 3, núm. 3 Esp., (2019)

Joao Andres Rodríguez Molina; Pedro Javier Chong Cevallos; Julio César Tixe Peralta; Rogelio Andrés Leyton Acuña

\section{RESUMEN}

En esta investigación se estudió los problemas relacionados a la dificultad respiratoria en los recién nacidos, así como las técnicas para diagnosticar el nivel de gravedad de la misma con el propósito de valorar la importancia del uso de la Escala de Silverman en la reducción de muertes ocasionadas por afecciones respiratorias. Se fundamentó en una investigación documental en el marco de una metodología de revisión. Se determinó en primera instancia que el síndrome de dificultad respiratoria es bastante común en los neonatos, más aún en aquellos que nacen de forma prematura además de que constituye un cuadro patológico muy amplio con causas variadas. En consecuencia, se encontró que la aplicación de la Escala de Silverman es utilizada para valorar los niveles de dificultad respiratoria considerando 5 criterios que pueden ser medidos sin perturbar al paciente. Sin embargo, se concluyó que a pesar de la facilidad de uso que tiene la escala, la confiabilidad de los puntajes obtenidos en ella no ha sido comprobada y existen pocos estudios de acceso libre que tengan el propósito de demostrar lo anterior.

Palabras claves: Neonatalidad; Dificultad respiratoria; Silverman; Causas de la dificultad respiratoria; Afecciones en neonatos. 


\section{Escala de Silverman en la dificultad respiratoria neonatal}

Vol. 3, núm. 3 Esp., (2019)

Joao Andres Rodríguez Molina; Pedro Javier Chong Cevallos; Julio César Tixe Peralta; Rogelio

Andrés Leyton Acuña

\section{ABSTRACT}

In this investigation we studied the problems related to respiratory distress in newborns, as well as techniques to diagnose the level of severity of the same in order to assess the importance of using the Silverman Scale in reducing deaths caused for respiratory conditions It was based on a documentary investigation within the framework of a review methodology. It was determined in the first instance that respiratory distress syndrome is quite common in neonates, even more so in those born prematurely, in addition to that it constitutes a very broad pathological picture with varied causes. Consequently, it was found that the application of the Silverman Scale is used to assess respiratory difficulty levels considering 5 criteria that can be measured without disturbing the patient. However, it was concluded that despite the ease of use of the scale, the reliability of the scores obtained in it has not been proven and there are few free access studies that have the purpose of demonstrating the above.

Keywords: Neonatality; Respiratory distress; Silverman; Causes of respiratory distress; Conditions in neonates. 


\section{Escala de Silverman en la dificultad respiratoria neonatal}

Vol. 3, núm. 3 Esp., (2019)

Joao Andres Rodríguez Molina; Pedro Javier Chong Cevallos; Julio César Tixe Peralta; Rogelio Andrés Leyton Acuña

\section{Introducción.}

En Ecuador, se registraron más de 290 mil neonatos durante todo el año 2018 (INEC (INEC - Ecuador, 2018). Estos, durante sus primeras semanas de vida experimentan un cambio de ambiente lo cual impulsa una serie de eventos críticos como la definición de conductas alimenticias y el aumento del riesgo a infecciones (Medline Plus, 2017).

Antes de nacer, los procesos fundamentales del feto como la respiración, la adquisición de nutrientes y la eliminación de desechos son llevados a cabo por la madre. Al nacer, estos procesos fundamentales deben adaptarse a las nuevas condiciones lo cual genera problemas debido a la gran transición que se requiere. Son muchos los factores que pueden intervenir, empeorando aún más la labor de supervivencia del neonato. Sin embargo, para estos bebes existen diversos cuidados especiales diseñados para mantener su salud durante esta dura etapa (Stanford Children's Health, 2019).

No obstante, los estudios relacionados a la reducción de la mortalidad neonatal están por debajo de los que al periodo posnatal respecta, lo cual, de continuar así, podría producir aumentos en la tasa de muertes de los neonatos a medida que avance el tiempo (Gomescoello, Romero, Lissette, \& Mendoza, 2018).

Uno de los problemas más comunes que experimentan los neonatos es el síndrome de dificultad respiratoria (SDR) determinado por una respiración anormal donde el paciente no puede realizar la correcta "oxigenación y eliminación del anhídrido carbónico” (López, Cruz, Hernández, \& Rentería, 2007). 


\section{Escala de Silverman en la dificultad respiratoria neonatal}

Vol. 3, núm. 3 Esp., (2019)

Joao Andres Rodríguez Molina; Pedro Javier Chong Cevallos; Julio César Tixe Peralta; Rogelio

Andrés Leyton Acuña

Es por ello que es importante diagnosticar rápidamente este síndrome durante las primeras horas de vida de los neonatos, para lo cual se puede utilizar la Escala de permite medir el grado de dificultad respiratoria (Shashidhar \& Shashidhar, 2016).

Esta escala fue diseñada por el Dr. William Silverman y Dorothy Andersen en 1956 y constituye una forma fácil, rápida y sencilla de aprender para evaluar los problemas respiratorios de los neonatos en una escala del 0 al 10 donde a medida que la puntuación aumenta es indicativo de mayor dificultad al respirar (Hedstrom, Gove, Mayock, \& Batra, 2018).

Sin embargo, a pesar del uso frecuente que se le da a la Escala de Silverman, hasta esta fecha existen pocos estudios que analicen la importancia de esta en la reducción de las tasas de mortalidad ocasionada por la dificultad respiratoria en los neonatos.

Por lo tanto, esta investigación tiene como propósito estudiar los riesgos relacionados al SDR y como a través de la Escala de Silverman puede detectarse a tiempo estas complicaciones para así aplicarse los cuidados necesarios, garantizando así la supervivencia de los neonatos.

\section{Materiales y Métodos.}

A octubre de 2019, se realizó una búsqueda estructurada de la literatura en las bases de datos SciELO, PubMed y Medline Plus con los términos "Escala de Silverman”, "Dificultad respiratoria neonatal”, "Síndrome de dificultad respiratoria”, "Neonatalidad en Ecuador", "Afecciones del neonato", "Utilidad de la Escala de Silverman" y 


\section{Escala de Silverman en la dificultad respiratoria neonatal}

Vol. 3, núm. 3 Esp., (2019)

Joao Andres Rodríguez Molina; Pedro Javier Chong Cevallos; Julio César Tixe Peralta; Rogelio Andrés Leyton Acuña

"Mortalidad por síndrome de dificultad respiratoria", tanto en español como en inglés. En función de determinar la importancia y validez de la Escala de Silverman en el diagnóstico de la diagnóstico de la dificultad respiratoria en neonatos, se incluyeron artículos científicos, estudio de cohorte, e-books, revisiones sistemáticas con o sin meta-análisis y demás documentos que a juicio del equipo investigador se encontraron revestidos de las características propias de las investigaciones de tipo científico-académica, publicados en lo que va de siglo XXI, salvo algunas excepciones. Se excluyeron casos y controles; series y reportes de casos; artículos de revisión narrativa; editoriales, y cartas al editor.

De forma secundaria, se realizó una búsqueda sin limitación cronológica para identificar información relevante diferente a lo expresado como términos de búsqueda anteriormente pero que sirvió para el desarrollo de la fundamentación teórica de la presente revisión. La inclusión o exclusión de cada una de las referencias en la presente revisión se definió por el consenso de los autores de este trabajo.

\section{Resultados.}

Durante los primeros momentos posteriores al nacimiento de un bebe, este puede experimentar diversas afecciones. Entre las más comunes se encuentran la distensión abdominal, lesiones durante el parto, el síndrome del bebé azul, tos, llanto excesivo, letargo y adormecimiento y por ultimo dificultad respiratoria (AAP, 2019). Es por esto que es de vital importancia realizar pruebas y análisis tanto visuales como químicos a los recién nacidos, a fin de detectar cualquier problema que presenten para así tomar los cuidados necesarios (Goya \& Soler, 2008). 


\section{Escala de Silverman en la dificultad respiratoria neonatal}

Vol. 3, núm. 3 Esp., (2019)

Joao Andres Rodríguez Molina; Pedro Javier Chong Cevallos; Julio César Tixe Peralta; Rogelio

Andrés Leyton Acuña

\section{Sindrome de dificultad respiratoria}

Una de las problemáticas más comunes que los neonatos experimentan es el síndrome de dificultad respiratoria la cual está compuesta por un conjunto de patologías que afectan la respiración y los mecanismos físicos relacionados a la misma. Para determinar su existencia es importante la realización de estudios radiológicos del tórax así como una exploración clínica (Coto, López, Fernández, Álvarez, \& Ibáñez, 2008).

La carencia de una sustancia denominada surfactante, que facilita la inflación de los pulmones con aire y protege a los alveolos de su colapso, compone una de las razones principales de la aparición de problemas al respirar debido a que esta sustancia tiende a escasear en bebes prematuros (Medline Plus, 2019).

Índice de dificultad respiratoria en recién nacidos

Basándose en datos de la UNICEF, López, Cruz, Hernández, \& Rentería (2007) señalan que los problemas respiratorios constituyen el $20 \%$ de las defunciones infantiles en los países en desarrollo y expresa que estadísticas originarias de 42 países apuntan que únicamente la mitad de los afectados se colocan en cuidados adecuados.

Goya \& Soler (2008) expresa que la severidad y ocurrencia de estos problemas es mayor cuando menos desarrollados se encuentra los neonatos

Causas de la dificultad respiratoria

Los partos por cesárea son los que producen con mayor frecuencia recién nacidos con 


\section{Escala de Silverman en la dificultad respiratoria neonatal}

Vol. 3, núm. 3 Esp., (2019)

Joao Andres Rodríguez Molina; Pedro Javier Chong Cevallos; Julio César Tixe Peralta; Rogelio Andrés Leyton Acuña

problemas respiratorios, esto es debido a que, durante un parto natural, químicos liberados estimulan la suspensión de la producción de sustancias encomendadas de la expulsión y reabsorción del líquido en los pulmones, como lo indica Rachel A \& Lewis MD (2006) analizado por López, Cruz, Hernández, \& Rentería (2007).

Los neonatos prematuros frecuentemente desarrollan este síndrome debido a una deficiencia de surfactante, mientras por otro lado los demás tienden a sufrirla a causa de una infección, aspiración de meconio, asfixia de nacimiento o por retener los fluidos en sus pulmones. Cabe señalar que existe una importante necesidad de herramientas que permitan diagnosticar correctamente este síndrome para así aplicar los cuidados debidos en el tiempo justo (Hedstrom, Gove, Mayock, \& Batra, 2018).

GD, J, B, F, \& A. (2008) expresa que la variedad de causas que pueden dar origen a problemas respiratorios es amplia. De forma resumida, existen diversas categorías en las cuales se pueden enmarcar, como lo son causas respiratorias, malformaciones, obstrucción vía aérea superior, causas cardiovasculares, causas infecciosas, causas metabólicas, causas hematológicas y causas neurológicas.

\section{Cuidados y tratamiento}

Si se detecta el síndrome de dificultad respiratoria en el neonato se deben efectuar los cuidados necesarios en unidades de cuidados intensivos. Es recomendable la monitorización suave de la temperatura, la frecuencia cardiaca y respiratoria, así como los niveles de PO2 y la pCO2 calculada a través de electrodo transcutáneo. Para pacientes de gravedad, se recomienda cuidados y mediciones más drásticas (Goya \& Soler, 2008). 


\section{Escala de Silverman en la dificultad respiratoria neonatal}

Vol. 3, núm. 3 Esp., (2019)

Joao Andres Rodríguez Molina; Pedro Javier Chong Cevallos; Julio César Tixe Peralta; Rogelio

Andrés Leyton Acuña

Algunos de los tratamientos y cuidados a los que son sometidos los neonatos que sufren de dificultad respiratoria son:

- Despejar las vías áreas, garantizando la respiración y circulación correcta del aire.

- Ofrecer oxigeno humidificado de forma asistida. Este debe ser administrado de forma cuidadosa a fin de impedir los problemas relacionados a los altos niveles del mismo.

- Sostenimiento de la correcta temperatura.

- Administración de fluidos y electrolitos.

- Mantenimiento adecuado de la hemoglobina.

- Suporte respiratorio dado en forma de presión aérea positive continua o ventilación obligada intermitente.

(Mathai, Raju, \& Kanitkar, 2007; Medline Plus, 2019).

Pruebas para detectar el síndrome de Dificultad Respiratoria

Luego del nacimiento, es importante llevar a cabo pruebas para evaluar la salud de los neonatos con la finalidad de detectar cualquier afección que puedan tener. A lo largo de la historia se han diseñados distintas pruebas para este propósito, entre las cuales están las siguientes. 


\section{Escala de Silverman en la dificultad respiratoria neonatal}

Vol. 3, núm. 3 Esp., (2019)

Joao Andres Rodríguez Molina; Pedro Javier Chong Cevallos; Julio César Tixe Peralta; Rogelio Andrés Leyton Acuña

\section{Puntuación de Apgar}

Se utiliza para evaluar la frecuencia cardiaca del recién nacido, así como su tono muscular y otros signos que ayudan a determinar si requiere atención adicional. Considera cinco criterios evaluándolos en una escala del 0 al 2, siendo este el máximo valor posible por criterio. El aspecto, el pulso, la irritabilidad, la actividad y la respiración son valorados durante la realización de esta prueba. Luego de sumar las puntuaciones obtenidas, se consideran a los neonatos que posean puntuaciones bajas como candidatos a recibir cuidados especiales. Cabe señalar que los puntajes obtenidos en esta prueba no predicen la salud a largo plazo del bebe, solo funciona para evaluar el estado del mismo para diagnosticar así si requiere de atención médica (The Nemours Foundation, 2019).

\section{Escala de Downes}

Basándose en el estudio de Wood DW, Downes JJ \& Locks HI (1972), Mathai, Raju, \& Kanitkar (2007) explica que este puntaje sirve para medir el nivel de dificultad respiratoria que presenta un neonato, tomado en cuenta 5 criterios y valorándolos en una escala del 0 al 2 . Por lo general, luego de sumar los valores obtenidos, se dice que un puntaje mayor a 6 es indicativo de dificultad al respirar. Los aspectos evaluados son:

- Frecuencia respiratoria, desde menor de 60 por minuto hasta más de 80 por minuto.

- Cianosis, desde su ausencia hasta con más de $40 \%$.

- Entrada de aire, desde una normal hasta una muy marcada. 


\section{Escala de Silverman en la dificultad respiratoria neonatal}

Vol. 3, núm. 3 Esp., (2019)

Joao Andres Rodríguez Molina; Pedro Javier Chong Cevallos; Julio César Tixe Peralta; Rogelio

Andrés Leyton Acuña

- Quejido respiratorio, desde ninguno hasta uno audible a la distancia.

- Retracciones subcostales, desde ninguna hasta una marcada.

(Mathai, Raju, \& Kanitkar, 2007).

El autor anterior añade que la Escala de Downes es bastante comprensiva y puede ser aplicada durante cualquier edad gestacional y condición.

\section{Escala de Silverman}

Toma el nombre de su creador el Dr. William Silverman quien dedicó gran parte de su vida a la investigación referente a la neonatología. También denominada como Puntuación de Dificultad Respiratoria, fue diseñada junto a su colaboradora Dorothy Andersen en 1956 para cuantificar los niveles de dificultad respiratoria que pueda sufrir un neonato. En la actualidad, las instalaciones con infraestructura de bajos recursos lo utilizan como una prueba objetiva, fácil de aprender, rápida de realizar y llevar a cabo sin necesidad de equipamientos costosos. Puede ser enseñada y llevada a cabo por personal con escaso entrenamiento médico y puede ser ejecutada sin invadir físicamente al paciente. Posee una larga historia de uso en complejos de bajos recursos, donde se utiliza para diagnosticar los problemas relacionados a la dificultad respiratoria. Es una valiosa herramienta para detectar niños que requieran cuidados especiales (Hedstrom, Gove, Mayock, \& Batra, 2018).

Analizando lo propuesto por Silverman WC \& Anderson DH (1956), Mathai, Raju, \& Kanitkar (2007) expone que esta prueba evalúa, en con una puntuación del 0 al 2, los siguientes 


\section{Escala de Silverman en la dificultad respiratoria neonatal}

Vol. 3, núm. 3 Esp., (2019)

Joao Andres Rodríguez Molina; Pedro Javier Chong Cevallos; Julio César Tixe Peralta; Rogelio Andrés Leyton Acuña

criterios:

- Retracción de la parte alta del pecho, desde sincronizada hasta asíncrona.

- Retracción de la parte baja del pecho, desde ninguna hasta marcada.

- Retracción xifoidea, desde ausente hasta marcada.

- Dilatación nasal, desde ninguna hasta marcada.

- Quejido respiratorio, desde ninguno hasta audible a la distancia.

(Mathai, Raju, \& Kanitkar, 2007)

Luego de sumar los valores obtenidos, se consideran como neonatos que sufren de dificultad respiratoria a aquellos que obtengan puntajes mayores a 6 .

\section{Comparación de la Escala de Silverman con la Escala de Downes}

A, PN, \& Jose (2016) señala que estas escalas son usadas comúnmente para el rápido diagnóstico de la dificultad respiratoria, así como su nivel de gravedad. Este nivel es importante en la toma de decisiones respecto al manejo del paciente, que puede incluir ventilación mecánica en algunos casos. La Escala de Downes es usada para evaluar tanto a los neonatos a término como a los prematuros mientras que la Escala de Silverman solo ha sido validada en la calificación de bebes pre término.

El mismo en su estudio aplicó una observación comparativa en una unidad de cuidados neonatales donde internos y enfermeras fueron entrenados en la aplicación de ambas escalas para 


\section{Escala de Silverman en la dificultad respiratoria neonatal}

Vol. 3, núm. 3 Esp., (2019)

Joao Andres Rodríguez Molina; Pedro Javier Chong Cevallos; Julio César Tixe Peralta; Rogelio

Andrés Leyton Acuña

luego utilizarlas en recién nacidos y dar así su opinión respecto a la dificultad de uso de las mismas, mientras simultáneamente un investigador realizaba la misma labor a fin de servir como mediador. En total participaron 20 internos y 20 enfermeras (40 participantes en total) y se determinó que la Escala de Downes, tiende a ser mejor que la Escala de Silverman en lo que a determinar la gravedad de dificultad respiratoria de los bebes prematuros respecta además de tener mayor eficacia y fiabilidad. Además, gracias a este estudio se dio a conocer que la Escala de Silverman se encuentra correlacionada con la mortalidad mientras que la Escala de Downes con parámetros de gasometría arterial.

Sin embargo, en Mathai, Raju, \& Kanitkar (2007), se expresa que, aunque la Escala de Silverman es mejor para recién nacidos pre término con la enfermedad de membrana hialina, la cual constituye, según GD, J, B, F, \& A. (2008), “el cuadro más significativo de dificultad respiratoria neonatal", la Escala de Downes es más amplia, además permite su aplicación durante cualquier edad gestacional y bajo cualquier condición.

Por otra parte, en el estudio de cohorte prospectivo de Hedstrom, Gove, Mayock, \& Batra (2018), donde se analizaron datos recolectados de una unidad de cuidados intensivos neonatales durante 12 meses sobre recién nacidos admitidos durante su primera hora de vida, excluyendo a aquellos que presentaran "intubación previa a la primera hora de vida, muerte durante las primeras 24 horas del nacimiento, diagnóstico de hipoxia encefalopatía isquémica o una condición neuromuscular primaria”, sus resultados dieron a conocer que, a pesar de que muchas instalaciones médicas de bajos recursos usan rangos de puntajes entre 3 a 6 de la Escala de Silverman para guiar las decisiones de iniciar o incrementar la asistencia respiratoria debido a la 


\section{Escala de Silverman en la dificultad respiratoria neonatal}

Vol. 3, núm. 3 Esp., (2019)

Joao Andres Rodríguez Molina; Pedro Javier Chong Cevallos; Julio César Tixe Peralta; Rogelio Andrés Leyton Acuña

relación que poseen, no fue posible verificar la fiabilidad de los puntajes obtenidos en relación al nivel de dificultad respiratoria, debido principalmente a problemas estadísticos y de registro.

\section{Conclusiones.}

Al examinar la información recolectada, se concluye que el diagnóstico adecuado del síndrome de dificultad respiratoria en los recién nacidos para tomar los cuidados necesarios a tiempo es de vital importancia debido a las altas tasas de mortalidad que se le atribuyen a este. Por otra parte, se revela el uso de la Escala de Silverman como una valiosa herramienta para predecir la necesidad de asistencia respiratoria en los neonatos, considerando que la eficacia de los puntajes obtenidos en esta escala en comparación a las pruebas de laboratorio más elaboradas y a otras escalas similares no ha sido comprobada o los resultados de los estudios relacionados no se encuentran disponibles al público, pero debido a su popularidad y su fácil aplicación, compone el método de diagnóstico adecuado en situaciones donde los aparatos de examinación costosos no se encuentran disponible.

\section{Bibliografía.}

AAP. (2019). Afecciones comunes de los recién nacidos. Obtenido de American Academy of Pediatrics: https://www.healthychildren.org/Spanish/ages-stages/baby/Paginas/commonconditions-in-newborns.aspx

Coto, G., López, J., Fernández, B., Álvarez, F., \& Ibáñez, A. (2008). Recién nacido a término con dificultad respiratoria: enfoque diagnóstico y terapéutico. Protocolos Diagnóstico Terapeúticos de la AEP: Neonatología(30), 285-305.

Gomescoello, M. E., Romero, A. E., Lissette, E. E., \& Mendoza, J. L. (2018). Causas y diagnóstico de sepsis tardía en neonatos. Revista Científica de Investigación actualización del mundo de las Ciencias, 3(2), 1086-1105. 


\section{Escala de Silverman en la dificultad respiratoria neonatal}

Vol. 3, núm. 3 Esp., (2019)

Joao Andres Rodríguez Molina; Pedro Javier Chong Cevallos; Julio César Tixe Peralta; Rogelio

Andrés Leyton Acuña

Goya, J. L., \& Soler, A. V. (2008). Síndrome de dificultad respiratoria. Protocolos Diagnóstico Terapeúticos de la AEP: Neonatología(31), 305-310.

Hedstrom, A. B., Gove, N. E., Mayock, D. E., \& Batra, M. (Mayo de 2018). Performance of the Silverman Andersen Respiratory Severity Score in predicting PCO2 and respiratory support in newborns: a prospective cohort study. Journal of Perinatology, 38(5), 505511.

INEC - Ecuador. (2018). Nacimientos y Defunciones. Obtenido de Instituto nacional de estadística y censos: https://www.ecuadorencifras.gob.ec/nacimientos_y_defunciones/

López, F. W., Cruz, Y. L., Hernández, G. G., \& Rentería, Y. B. (2007). Factores de riesgo del Síndrome Dificultad Respiratoria de origen pulmonar en el recién nacido. Revista Cubana Enfermer, 23(3), 1-15.

Mathai, S., Raju, U., \& Kanitkar, M. (2007). Management of Respiratory Distress in the Newborn. Medical journal, Armed Forces India, 63(3), 269-272.

Medline Plus. (18 de 10 de 2017). Neonato. Obtenido de MedlinePlus: https://medlineplus.gov/spanish/ency/article/002271.htm

Medline Plus. (4 de Abril de 2019). Síndrome de dificultad respiratoria neonatal. Obtenido de MedlinePlus: https://medlineplus.gov/spanish/ency/article/001563.htm

Shashidhar, A., \& Shashidhar, P. (2016). Downes Score vs Silverman Anderson Score for Assessment of Respiratory Distress in Preterm Newborns. Pediatric Oncall Journal, 13(3), 66-68.

Stanford Children's Health. (2019). Unidad neonatal de cuidados intensivos (UNCI). Obtenido de Stanford Children's Health: https://www.stanfordchildrens.org/es/topic/default?id=theneonatal-intensive-care-unit-nicu-90-P05498

The Nemours Foundation. (2019). ¿Qué es la puntuación de Apgar? Obtenido de KidsHealth: https://kidshealth.org/es/parents/apgar-esp.html

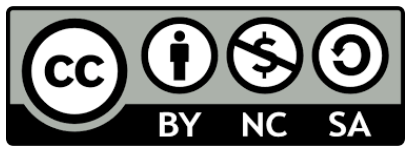

RECONOCIMIENTO-NOCOMERCIAL-COMPARTIRIGUAL

CC BY-NC-SA

ESTA LICENCIA PERMITE A OTROS ENTREMEZCLAR, AJUSTAR Y CONSTRUIR A PARTIR DE SU OBRA CON FINES NO

COMERCIALES, SIEMPRE Y CUANDO LE RECONOZCAN LA AUTORÍA Y SUS NUEVAS CREACIONES ESTÉN BAJO UNA LICENCIA CON LOS MISMOS TÉRMINOS. 